\title{
On the Relationship Between Complex Potentials and Strings of Projection Operators
}

\author{
J.J.Halliwell and J.M.Yearsley \\ Blackett Laboratory \\ Imperial College \\ London $S W 72 B Z$ \\ $U K$
}

(Dated: March 22, 2022)

\begin{abstract}
It is of interest in a variety of contexts, and in particular in the arrival time problem, to consider the quantum state obtained through unitary evolution of an initial state regularly interspersed with periodic projections onto the positive $x$-axis (pulsed measurements). Echanobe, del Campo and Muga have given a compelling but heuristic argument that the state thus obtained is approximately equivalent to the state obtained by evolving in the presence of a certain complex potential of stepfunction form. In this paper, with the help of the path decomposition expansion of the associated propagators, we give a detailed derivation of this approximate equivalence. The propagator for the complex potential is known so the bulk of the derivation consists of an approximate evaluation of the propagator for the free particle interspersed with periodic position projections. This approximate equivalence may be used to show that to produce significant reflection, the projections must act at time spacing less than $\hbar / E$, where $E$ is the energy scale of the initial state.

PACS numbers:
\end{abstract}




\section{INTRODUCTION}

A number of physically interesting situations in quantum theory concern the question of what happens to an initial quantum state $|\psi\rangle$ which is acted on by a sequence of projection operators interspersed with unitary evolution for time $\epsilon$,

$$
\left|\psi_{P}(\tau)\right\rangle=e^{-i H \epsilon} P e^{-i H \epsilon} \ldots P e^{-i H \epsilon}|\psi\rangle
$$

(Here, there are $n$ projection operators and $\tau=(n+1) \epsilon$ and we use units in which $\hbar=1$ ).

Such an object describes pulsed measurements but it also crops up in the decoherent histories approach to quantum theory, where the right-hand side is the amplitude for a quantummechanical history [1-6].

We are interested in the specific case of a free particle with $P$ taken to be the projection onto the positive $x$-axis, $P=\theta(\hat{x})$. For sufficiently small $\epsilon$, Eq.(1.1) is then a candidate for the amplitude to remain in $x>0$ during the time interval $[0, \tau]$, an object that is of interest in the arrival time problem [7-10].

It is of interest to explore the properties of this amplitude for a range of values of the time spacing $\epsilon$. It is known that as $\epsilon \rightarrow 0$, we approach the Zeno limit, in which the state becomes entirely confined to the Hilbert subspace of states with support only in $x>0$, so that an incoming wave packet from the right is totally reflected [11-13]. However, it is of greater physical interest to explore the regime of non-zero $\epsilon$, in which the system is monitored sufficiently well to get some idea of whether the particle is in $x>0$, yet not monitored so much that an incoming state is significantly reflected at $x=0$. An important question in this regime is to determine the value of $\epsilon$ for which reflection becomes significant. For an initial state with energy width $\Delta H$, a timescale held to be significant is the Zeno time,

$$
t_{Z}=\frac{1}{\Delta H}
$$

which is the timescale on which the state becomes significantly different from its initial value under unitary evolution [11]. For a wave packet of momentum $p$ and spatial width $\sigma$, the Zeno time is of order $m \sigma / p$ which is the timescale on which the wave packet crosses the origin. This indicates that the Zeno time for wave packets is an essentially classical timescale and, in Eq.(1.1), relates only to the rate of removal of probability through projection. By contrast, reflection in Eq.(1.1) arises as a result of the increase in uncertainty in momentum 
resulting from position projection, an obviously quantum process, so one would expect it to have a different timescale, which could be much shorter than then Zeno time. It would be of interest to compute this timescale. One reason it is important is that there appears to be interesting physics very close to the Zeno limit [14-16].

A significant result in this area is due to Echanobe, del Campo and Muga, who claimed that for finite $\epsilon$ the string of projection operators in Eq.(1.1) is approximately equivalent to evolution in the presence of a complex potential [14]. That is,

$$
e^{-i H \epsilon} P e^{-i H \epsilon} \cdots P e^{-i H \epsilon} \approx \exp \left(-i H \tau-V_{0} \theta(-x) \tau\right)
$$

They assert that this result is valid if, for a given $\epsilon, V_{0}$ is chosen such that two conditions

$$
\begin{aligned}
& V_{0} \epsilon \gg 1 \\
& V_{0} \gg \Delta H
\end{aligned}
$$

are satisfied. This is a very useful result since Eq.(1.1) is not easy to evaluate analytically but the Schrödinger equation with a complex step potential in Eq.(1.3) can be solved straightforwardly. Furthermore, such complex potentials have been studied extensively in the literature and can often be linked to particular detection methods [17].

Given the connection Eq.(1.3), one can determine the conditions under which reflection becomes important. Known results on scattering with the complex potential in Eq.(1.3) show that, for an incoming state with energy scale $E$, reflection becomes significant when $V_{0}>E[8,9]$. Reflection is avoided, therefore, when $V_{0} \ll E$, or equivalently, from Eq.(1.4), when

$$
\epsilon \gg \frac{1}{E}
$$

This is much less than the Zeno time for a state strongly peaked in energy. There is therefore an interesting regime, namely

$$
\frac{1}{E} \ll \epsilon \ll \frac{1}{\Delta H}
$$

in which the projections in Eq.(1.1) are sufficiently frequent to have a significant effect on the system, yet not so frequent that there is significant reflection.

Eq.(1.6) is a very useful result, but it has been derived on the basis of the claimed approximate relationship Eq.(1.3). The derivation of Eq.(1.3) given by Echanobe et al is very plausible (and was also hinted at by Allcock [8]), but it is rather heuristic, and the 
deduced connection Eq.(1.4) between $V_{0}$ and $\epsilon$ is rather loose. There is therefore considerable scope for a more detailed and substantial derivation.

The purpose of this paper is to give a more substantial derivation of the equivalence Eq.(1.3) and to deduce a more precise relationship between $V_{0}$ and $\epsilon$. We will do this by computing the configuration space propagators associated with each side of Eq.(1.3) and

show that they are approximately equal in certain regimes. The propagator associated with the complex potential is in fact known already, so the bulk of the work consists of an approximate evaluation of the propagator associated with pulsed measurements, the lefthand side of Eq.(1.3).

This is certainly not a rigorous mathematical proof of Eq.(1.3), involving operator norms, error bounds and the like, but a theoretical physicists style of proof involving the approximate evaluation of propagators. A rigorous proof would certainly be of interest to construct and the work described in this paper may give some hints in that direction.

We begin in Section 2 with a brief summary of the derivation of Echanobe et al, with a small extension of it that turns out to be important and yields an equality relating $V_{0}$ and $\epsilon$, thereby improving on Eq.(1.4). We then in Section 3 give a detailed formulation of the problem and how we solve it. The key idea is to use the path decomposition expansion in which the propagators associated with each side of Eq.(1.3) are factored across the surface $x=0$ [18-20]. The problem of proving the equivalence Eq.(1.3) thereby reduces to proving it for propagation between points at $x=0$ for different times. Since the propagator for the complex potential is known, the main work is to evaluate this propagator for pulsed measurements. This is actually rather difficult to do directly, but a good approximate analytic expression can be obtained for it by attacking the problem from a number of different angles. This is described in Sections 4, 5, 6 and 7. In Section 8 we give a detailed discussion of the timescales involved for the approximations to be valid. We summarize and conclude in Section 9.

\section{REVIEW AND EXTENSION OF EARLIER WORK}

We first review and extend the derivation of Echanobe et al [14]. They first note that

$$
\exp \left(-V_{0} \theta(-\hat{x}) \epsilon\right)=P+e^{-V_{0} \epsilon} \bar{P}
$$


where, recall $P=\theta(\hat{x})$ and $\bar{P}=1-P$. It follows that

$$
P=\theta(\hat{x}) \approx \exp \left(-V_{0} \theta(-\hat{x}) \epsilon\right)
$$

as long as the parameter

$$
\alpha=V_{0} \epsilon
$$

is sufficiently large that

$$
e^{-\alpha} \ll 1
$$

Eq.(1.3) then follows from the approximate equivalence,

$$
\exp (-i H \epsilon) \exp \left(-V_{0} \theta(-\hat{x}) \epsilon\right) \approx \exp \left(-i H \epsilon-V_{0} \theta(-\hat{x}) \epsilon\right)
$$

which will hold as long as

$$
V_{0} \epsilon^{2}|\langle[H, \theta(-\hat{x})]\rangle| \ll 1
$$

where the average is taken in the initial state. Echanobe et al put an upper bound on the left-hand side using the Schrödinger-Robertson inequality and Eq.(2.6) may then be written in either of the two equivalent forms

$$
\alpha^{2} \Delta H \ll V_{0}
$$

or

$$
\alpha \epsilon \ll \frac{1}{\Delta H}
$$

which implies that the time between projections is much less than the Zeno time, the typical timescale on which the state undergoes significant change [11]. (The conditions Eqs.(2.3), (2.4), (2.7) are a more precise version of the originally stated conditions Eqs.(1.4), (1.5)).

Since the parameter $\alpha$ need only satisfy an inequality, Eq.(2.4), the relationship Eq.(2.3) between $\epsilon$ and $V_{0}$ is not uniquely determined. However, it turns out that the above derivation can be extended somewhat to give an equality between $\epsilon$ and $V_{0}$. This turns out to be relevant to the more substantial derivation given in the rest of this paper.

The above result may be written,

$$
\exp \left(-i H \epsilon-V_{0} \theta(-\hat{x}) \epsilon\right) \approx \exp (-i H \epsilon) P
$$

under the conditions given above. Now suppose $\alpha$ is an integer and write $\epsilon=\alpha \epsilon^{\prime}$, where

$$
V_{0} \epsilon^{\prime}=1
$$


Then, since $P=P^{2}$, we may approximate Eq.(2.9) by

$$
\begin{aligned}
\exp \left(-i H \epsilon-V_{0} \theta(-\hat{x}) \epsilon\right) & \approx e^{-i H \epsilon^{\prime}} P e^{-i H \epsilon^{\prime}} P \cdots e^{-i H \epsilon^{\prime}} P \\
& =\left(e^{-i H \epsilon^{\prime}} P\right)^{\alpha}
\end{aligned}
$$

as long as the contribution from the commutator terms between $e^{-i H \epsilon^{\prime}}$ and $P$ is sufficiently small. There will be of order $\alpha^{2}$ such terms, hence the error in this approximation is of order $\alpha^{2} \epsilon^{\prime} \Delta H$ and from Eq.(2.7), this error is much less than 1. The key point here is that even the longer timescale $\epsilon$ is still much less than the Zeno time and, since nothing changes on this timescale, there is essentially no difference between Eqs.(2.9) and (2.11).

We therefore see that the desired result Eq.(1.3) actually holds for much smaller time steps, defined by the equality Eq.(2.10). This is different to the original claim of Echanobe et al, since they require the inequality Eq.(1.4). However, the above argument shows that the restriction Eq.(1.4) is in fact stronger than necessary and in the following pages our more detailed derivation will show that Eq.(1.3) does indeed hold with a timespacing of order $1 / V_{0}$.

\section{DETAILED FORMULATION OF THE PROBLEM}

In this paper, we will prove the relationship Eq.(1.3) in a much more substantial way by proving the approximate equivalence of the propagators

$$
\begin{aligned}
& g_{V}\left(x_{1}, \tau \mid x_{0}, 0\right)=\left\langle x_{1}\left|\exp \left(-i H \tau-V_{0} \theta(-x) \tau\right)\right| x_{0}\right\rangle \\
& g_{P}\left(x_{1}, \tau \mid x_{0}, 0\right)=\left\langle x_{1}\left|e^{-i H \epsilon_{n}} P e^{-i H \epsilon} \ldots e^{-i H \epsilon} P e^{-i H \epsilon_{0}}\right| x_{0}\right\rangle
\end{aligned}
$$

for some relationship between the parameters $\epsilon$ and $V_{0}$, to be determined. Note that in Eq.(3.2), we have chosen the initial and final time spacings to be $\epsilon_{0}$ and $\epsilon_{n}$, with

$$
\tau=(n-1) \epsilon+\epsilon_{0}+\epsilon_{n}
$$

This turns out to be necessary for the most general proof of Eq.(1.3). For the special case $\epsilon_{n}=\epsilon=\epsilon_{0}$, we may also write

$$
g_{P}\left(x_{1}, \tau \mid x_{0}, 0\right)=\left\langle x_{1}\left|e^{-i H \tau} P(n \epsilon) \cdots P(2 \epsilon) P(\epsilon)\right| x_{0}\right\rangle
$$


Each of the above propagators may be represented by a path integral,

$$
\begin{aligned}
& g_{V}\left(x_{1}, \tau \mid x_{0}, 0\right)=\int \mathcal{D} x \exp \left(i \int_{0}^{\tau} d t\left[\frac{1}{2} m \dot{x}^{2}+i V_{0} \theta(-x)\right]\right) \\
& g_{P}\left(x_{1}, \tau \mid x_{0}, 0\right)=\int_{P} \mathcal{D} x \exp \left(i \int_{0}^{\tau} d t \frac{1}{2} m \dot{x}^{2}\right)
\end{aligned}
$$

where in both cases the paths are from $x(0)=x_{0}$ to $x(\tau)=x_{1}$ and in the second case, Eq.(3.6), $P$ denotes that the paths are restricted to be in the positive $x$-axis at times $t=\epsilon_{0}+(k-1) \epsilon, k=1,2 \cdots n$.

A closely related object that will be important is the restricted propagator,

$$
g_{r}\left(x_{1}, \tau \mid x_{0}, 0\right)=\int_{x(t)>0} \mathcal{D} x \exp \left(i \int_{0}^{\tau} d t \frac{1}{2} m \dot{x}^{2}\right)
$$

where again the paths are from $x(0)=x_{0}$ to $x(\tau)=x_{1}$ but with $x(t)>0$ for all times in $[0, \tau]$. This is clearly equivalent to $g_{P}$ in Eq.(3.6), in the limit $n \rightarrow \infty, \epsilon \rightarrow 0$ with $\tau$ constant. If we take the same limit in the equivalent expression for $g_{P}$, Eq.(3.2), one obtains the following convenient operator form of the restricted propagator:

$$
g_{r}(\tau, 0)=P \exp (-i P H P \tau)
$$

The restricted propagator satisfies the Schrödinger equation in $x>0$ subject to the boundary conditions that it vanishes when either end of the propagator sits on $x=0$. For the free particle, considered here, one can easily solve for the restricted propagator using the method of images and the result is

$$
\begin{aligned}
g_{r}\left(x_{1}, \tau \mid x_{0}, 0\right) & =\left(\frac{m}{2 \pi i \tau}\right)^{1 / 2} \theta\left(x_{1}\right) \theta\left(x_{0}\right) \\
& \times\left[\exp \left(\frac{i m\left(x_{1}-x_{0}\right)^{2}}{2 \tau}\right)-\exp \left(\frac{i m\left(x_{1}+x_{0}\right)^{2}}{2 \tau}\right)\right]
\end{aligned}
$$

The restricted propagator, in any of the above forms, describes the regime of "Zeno dynamics", in which all states are confined entirely to the Hilbert subspace of states with support only in $x>0[13]$.

The restricted propagator plays an important role here since not only does $g_{P} \rightarrow g_{r}$ in the limit $n \rightarrow \infty, \epsilon \rightarrow 0$ with $\tau$ constant, but also $g_{V} \rightarrow g_{r}$ as $V_{0} \rightarrow \infty$. It follows that $g_{V}$ and $g_{P}$ become arbitrarily close to each other for sufficiently large $V_{0}$ and $n$, since they both tend to the same limit. This is the underlying reason why we expect the approximate equivalence Eq.(1.3) should hold. 
The propagators $g_{P}$ and $g_{V}$ may be decomposed using the path decomposition expansion (PDX), in which a propagator, regarded as a sum over paths, is split into propagation corresponding to paths (or sections of paths) entirely in $x>0$, paths entirely in $x<0$ and paths starting and ending on the boundary [18-20]. There are two cases to consider.

We consider first the case in which the initial and final points are on the same side of the surface in $x>0$. The set of paths from initial to final point may be partitioned into paths that never cross the origin, represented by the restricted propagator $g_{r}$, and paths that always cross. The paths that cross have a first crossing at $t_{1}$ and a last crossing at $t_{2}$. The corresponding PDX for any propagator $g$ has the form

$$
\begin{aligned}
g\left(x_{1}, \tau \mid x_{0}, 0\right) & =\left.\left.\frac{1}{4 m^{2}} \int_{0}^{\tau} d t_{2} \int_{0}^{t_{2}} d t_{1} \frac{\partial g_{r}}{\partial x}\left(x_{1}, \tau \mid x, t_{2}\right)\right|_{x=0} g\left(0, t_{2} \mid 0, t_{1}\right) \frac{\partial g_{r}}{\partial x}\left(x, t_{1} \mid x_{0}, 0\right)\right|_{x=0} \\
& +g_{r}\left(x_{1}, \tau \mid x_{0}, 0\right)
\end{aligned}
$$

This is depicted in Figure 1. The first term in this expression, describing paths which cross the origin, contains the derivative of $g_{r}$ because this corresponds to paths which never cross $x=0$ but end or begin at $x=0$ (recalling that $g_{r}$ itself vanishes if either end is on $x=0$ ) [18].

Eq.(3.10) holds for both $g_{P}$ and $g_{V}$, but the restricted part $g_{r}$ is the same in each case and equal to Eq.(3.9) above, since the restriction on paths defining $g_{P}$ and the presence of the complex potential in $g_{V}$ in Eqs.(3.5), (3.6) are both redundant if $x(t)>0$. Eq.(3.10) may therefore be simplified using

$$
\left.\frac{\partial g_{r}}{\partial x}\left(x, t_{1} \mid x_{0}, 0\right)\right|_{x=0}=2 \frac{\partial g_{f}}{\partial x}\left(0, t_{1} \mid x_{0}, 0\right)
$$

which follows from Eq.(3.9), where $g_{f}$ denotes the free particle propagator.

It follows from the above that $g_{P}$ and $g_{V}$ could differ only in terms of their propagation along $x=0$, hence to prove the approximate equivalence of the propagators Eqs.(3.1), (3.2), we need to prove that

$$
g_{P}\left(0, t_{2} \mid 0, t_{1}\right) \approx g_{V}\left(0, t_{2} \mid 0, t_{1}\right)
$$

Note that, unlike $g_{V}\left(0, t_{2} \mid 0, t_{1}\right), g_{P}\left(0, t_{2} \mid 0, t_{1}\right)$ is not covariant under time translation, that is,

$$
g_{P}\left(0, t_{2} \mid 0, t_{1}\right) \neq g_{P}\left(0, t_{2}-t_{1} \mid 0,0\right)
$$

although it has an approximate covariance on timescales much greater than $\epsilon$, as we will see below. 
The second case is that in which the initial and final points are on opposite sides of the surface, $x_{1}<0$ and $x_{0}>0$. In this case we have for $g_{V}$,

$$
\begin{aligned}
g_{V}\left(x_{1}, \tau \mid x_{0}, 0\right) & =\left.\frac{1}{4 m^{2}} \int_{0}^{\tau} d t_{2} e^{-V_{0}\left(\tau-t_{2}\right)} \int_{0}^{t_{2}} d t_{1} \frac{\partial g_{r}}{\partial x}\left(x_{1}, \tau \mid x, t_{2}\right)\right|_{x=0} \\
& \times\left. g_{V}\left(0, t_{2} \mid 0, t_{1}\right) \frac{\partial g_{r}}{\partial x}\left(x, t_{1} \mid x_{0}, 0\right)\right|_{x=0}
\end{aligned}
$$

since the last section of the paths is in $x<0$ where the complex potential acts. For $g_{P}$, if $x_{1}<0$, since the paths must be in $x>0$ at the given discrete set of times, the last crossing time $t_{2}$ cannot be less than the last time $\tau-\epsilon_{n}$ at which the projectors act, hence

$$
\begin{aligned}
g_{P}\left(x_{1}, \tau \mid x_{0}, 0\right) & =\left.\frac{1}{4 m^{2}} \int_{\tau-\epsilon_{n}}^{\tau} d t_{2} \int_{0}^{t_{2}} d t_{1} \frac{\partial g_{r}}{\partial x}\left(x_{1}, \tau \mid x, t_{2}\right)\right|_{x=0} \\
& \times\left. g_{P}\left(0, t_{2} \mid 0, t_{1}\right) \frac{\partial g_{r}}{\partial x}\left(x, t_{1} \mid x_{0}, 0\right)\right|_{x=0}
\end{aligned}
$$

Again we will have to prove that Eq.(3.12) holds, but these two expressions Eqs.(3.14), (3.15) also differ in the form of the $t_{2}$ integral. However, since $V_{0} \epsilon \approx 1$, the form of the exponential in Eq.(3.14) effectively squeezes $t_{2}$ to lie approximately within $\epsilon$ of $\tau$, so we have

$$
\int_{0}^{\tau} d t_{2} e^{-V_{0}\left(\tau-t_{2}\right)} \approx \int_{\tau-\epsilon_{n}}^{\tau} d t_{2}
$$

and Eqs.(3.14) and (3.15) are approximately the same.

We see that both cases reduce to proving Eq.(3.12). The propagator along the boundary for the complex potential is known [21], and is given by

$$
\begin{aligned}
g_{V}(0, t \mid 0,0) & =\left(\frac{m}{2 \pi i t}\right)^{1 / 2} \frac{\left(1-e^{-V_{0} t}\right)}{V_{0} t} \\
& :=\left(\frac{m}{2 \pi i t}\right)^{1 / 2} f_{V}(t) .
\end{aligned}
$$

The main purpose of the remainder of this paper is to calculate the propagator with projection operators along the boundary,

$$
g_{P}(0, \tau \mid 0,0)=\left\langle 0\left|e^{-i H \epsilon_{n}} P e^{-i H \epsilon} \cdots e^{-i H \epsilon} P e^{-i H \epsilon_{0}}\right| 0\right\rangle
$$

and show that the approximation Eq.(3.12) holds, under conditions to be determined. (Here, $|0\rangle$ denotes a position eigenstate $|x\rangle$ at $x=0)$.

In what follows, our main result is to show that, to a good approximation,

$$
g_{P}(0, t \mid 0,0) \approx\left(\frac{m}{2 \pi i t}\right)^{1 / 2} f_{P}(t)
$$


where $f_{P}(t)$ is a kind of saw-tooth function - a piecewise linear function with peaks immediately followed by troughs at $t_{k}=\epsilon_{0}+(k-1) \epsilon, k=1,2, \cdots$. Approaching $t_{k}$ from below, there is a peak of value

$$
f_{P}\left(t_{k}\right)=\frac{1}{k+1}
$$

and approaching $t_{k}$ from above there is a trough of half that size. That is

$$
f_{P}(t)=\frac{\left(t-t_{k-1}\right)}{(k+1) \epsilon}+\frac{\left(t_{k}-t\right)}{2 k \epsilon} \quad \text { for } \quad t_{k-1} \leq t<t_{k}, \quad k=2,3 \cdots
$$

and

$$
f_{P}(t)=1 \quad \text { for } \quad 0 \leq t<\epsilon_{0}
$$

The functions $f_{P}(t)$ and $f_{V}(t)$ are shown in Figure 2. We see that $f_{P}(t)$ oscillates with period $\epsilon$ about $f_{V}(t)$, as long as we choose $V_{0}$ so that $f_{V}(t)$ lies between the peaks and troughs of $f_{P}(t)$. That is, for large $k$, we require that

$$
\frac{1}{2 k}<\frac{1}{V_{0} t}<\frac{1}{k}
$$

Since $t \approx k \epsilon, f_{V}(t)$ will lie approximately midway between the peaks and troughs of $f_{P}(t)$ if

$$
V_{0} \epsilon \approx \frac{4}{3}
$$

Recalling that the propagator is attached through the PDX Eq.(3.10) to an initial state, the oscillations, and hence the differences between $g_{P}$ and $g_{V}$, will be smoothed out as long as $\epsilon$ is chosen to be smaller than the timescale of variation of the initial state. With some qualifications (discussed further in Section 8), this timescale is the Zeno time, $1 / \Delta H$. The desired approximation Eq.(3.12) will therefore hold in a time-averaged sense, and we thus have significant agreement with the extended version of the original argument of Echanobe et al described in Section 2.

In the following sections, we evaluate Eq.(3.18) and confirm the form Eq.(3.21) of $f_{P}(t)$. In Section 4 we evaluate Eq.(3.18) exactly for the cases of one, two and three projections. We also show why in general the troughs of $f_{P}(t)$ are exactly half the size of the peaks by considering the limit $\epsilon_{n} \rightarrow 0$ of Eq.(3.18). In Section 5, we use a lattice method to derive the magnitude of the peaks of $f_{P}(t)$ for large $k$. These results are substantiated in Section 6, where we use an S-matrix expansion to derive some exact results for a certain time-averaged version of Eq.(3.18). In Section 7, we fill in some of the gaps in these regimes and approximations by computing Eq.(3.18) using numerical methods. 
We will find in the numerical and analytic solutions that the function interpolating between the troughs and peaks of $f_{P}(t)$ is not in fact a linear function in general. It is interesting however, that, because of the slow time variation of the initial state in comparison to $\epsilon$, the precise form of this function turns out to be unimportant, and this is what makes the problem more tractable than one might expect. All that is important is the location of the peaks and troughs of the saw-tooth function and the period of their oscillation. It is this separation of timescales that also restores an approximate time translation covariance to $g_{P}$, even though it does not hold exactly in general, Eq.(3.13).

\section{EXACT ANALYTIC RESULTS}

In this section we carry out an exact evaluation of Eq.(3.18) for the case of one, two and three projections. For simplicity, we take the initial time interval $\epsilon_{0}$ to be $\epsilon$ and for the case of three projections we are able to carry out the calculation only in when the final time $\epsilon_{n}=\epsilon$. We thus compute the objects

$$
g_{P}(0, t \mid 0,0)= \begin{cases}\left\langle 0\left|e^{-i H t}\right| 0\right\rangle, & \text { if } 0 \leq t<\epsilon \\ \left\langle 0\left|e^{-i H(t-\epsilon)} P e^{-i H \epsilon}\right| 0\right\rangle, & \text { if } \epsilon \leq t<2 \epsilon \\ \left\langle 0\left|e^{-i H(t-2 \epsilon)} P e^{-i H \epsilon} P e^{-i H \epsilon}\right| 0\right\rangle, & \text { if } 2 \epsilon \leq t<3 \epsilon \\ \left\langle 0\left|e^{-i H \epsilon} P e^{-i H \epsilon} P e^{-i H \epsilon} P e^{-i H \epsilon}\right| 0\right\rangle, & \text { if } t=4 \epsilon\end{cases}
$$

We will show that

$$
g_{P}(0, t \mid 0,0)= \begin{cases}\left(\frac{m}{2 \pi i t}\right)^{\frac{1}{2}} & \text { if } 0 \leq t<\epsilon \\ \frac{1}{2}\left(\frac{m}{2 \pi i t}\right)^{\frac{1}{2}} & \text { if } \epsilon \leq t<2 \epsilon \\ \frac{1}{4}\left(1+\frac{2}{\pi} \operatorname{ArcTan}[(t-2 \epsilon) / t]^{\frac{1}{2}}\right)\left(\frac{m}{2 \pi i t}\right)^{\frac{1}{2}} & \text { if } 2 \epsilon \leq t<3 \epsilon \\ \frac{1}{4}\left(\frac{m}{2 \pi i t}\right)^{\frac{1}{2}} & \text { if } t=4 \epsilon\end{cases}
$$

thereby confirming the approximate form of the saw-tooth function, Eq.(3.21), for the first few projections. These expressions all have the property that the value of $g_{P}(0, t \mid 0,0)$ drops to half its value immediately after a projection and we show why this is true in general at the end of this section.

To compute Eq.(4.1) it turns out to be useful to consider more general objects, in which 
the projections may be $P, \bar{P}$ or the identity. We therefore consider the object

$$
\begin{aligned}
g_{P, C}(0, t \mid 0,0) & =\int_{C} d x_{1} \ldots d x_{n}\left(\frac{m}{2 \pi i \epsilon}\right)^{n / 2}\left(\frac{m}{2 \pi i(t-n \epsilon)}\right)^{1 / 2} \\
& \times \exp \left(\frac{i m x_{1}^{2}}{2 \epsilon}+\frac{i m}{2 \epsilon} \sum_{k=1}^{n-1}\left(x_{k}-x_{k+1}\right)^{2}+\frac{i m x_{n}^{2}}{2(t-n \epsilon)}\right)
\end{aligned}
$$

Here $C=\{+,-, 0 \ldots\}$ symbolically stands for the integration ranges of the $x_{k}$, eg $C=$ $\{+,-, 0 \ldots\}$ means $0<x_{1}<\infty,-\infty<x_{2}<0,-\infty<x_{3}<\infty$ etc. Performing a Wick rotation, $\epsilon \rightarrow-i \epsilon$ and changing variables yields

$$
\begin{aligned}
g_{P, C}(0,-i t \mid 0,0) & =\left(\frac{m}{2 \pi}\right)^{1 / 2} \int_{C} \frac{d y_{1} \ldots d y_{n}}{(\pi \epsilon)^{n / 2}(t-n \epsilon)^{1 / 2}} \\
& \times \exp \left(-\frac{y_{1}^{2}}{\epsilon}-\sum_{k=1}^{n-1} \frac{\left(y_{k}-y_{k+1}\right)^{2}}{\epsilon}-\frac{y_{n}^{2}}{(t-n \epsilon)}\right) \\
& :=\left(\frac{m}{2 \pi}\right)^{1 / 2} T_{C}(\epsilon, t)
\end{aligned}
$$

The cases of no projection and one projection are trivially evaluated, and we easily obtain the first two equations in Eq.(4.2). For the case of two projections, we need to evaluate the object

$$
T_{++}\left(\epsilon_{1}, \epsilon_{2}, \epsilon_{3}\right)=\int_{0}^{\infty} \frac{d y_{1} d y_{2}}{\pi \sqrt{\epsilon_{1} \epsilon_{2} \epsilon_{3}}} \exp \left(-\frac{y_{1}^{2}}{\epsilon_{1}}-\frac{\left(y_{1}-y_{2}\right)^{2}}{\epsilon_{2}}-\frac{y_{2}^{2}}{\epsilon_{3}}\right)
$$

(a slight generalization of the $T_{C}$ defined in Eq.(4.5)). Changing variables to $r=y_{1}, y=$ $y_{2} / y_{1}$ gives,

$$
\begin{aligned}
T_{++}\left(\epsilon_{1}, \epsilon_{2}, \epsilon_{3}\right) & =\int_{0}^{\infty} \frac{d r d y}{\pi \sqrt{\epsilon_{1} \epsilon_{2} \epsilon_{3}}} r \exp \left(-r^{2}\left\{\frac{1}{\epsilon_{1}}+\frac{(1-y)^{2}}{\epsilon_{2}}+\frac{y^{2}}{\epsilon_{3}}\right\}\right) \\
& =\int_{0}^{\infty} \frac{d y}{2 \pi} \frac{1}{\left(\epsilon_{2} \epsilon_{3}+\epsilon_{1} \epsilon_{3}(1-y)^{2}+\epsilon_{1} \epsilon_{2} y^{2}\right)} \\
& =\int_{0}^{\infty} \frac{d y}{2 \pi} \frac{1}{a+b y+c y^{2}}
\end{aligned}
$$

Where $a=\epsilon_{3}\left(\epsilon_{1}+\epsilon_{2}\right), b=-2 \epsilon_{1} \epsilon_{3}, c=\epsilon_{1}\left(\epsilon_{2}+\epsilon_{3}\right)$. Now use the change of variables,

$$
u=\frac{b}{2 \sqrt{c}}+\sqrt{c} y
$$

to obtain

$$
T_{++}\left(\epsilon_{1}, \epsilon_{2}, \epsilon_{3}\right)=\frac{1}{2 \pi} \sqrt{\frac{\epsilon_{2}}{\epsilon_{1} \epsilon_{3} c}} \int_{\frac{b}{2 \sqrt{c}}}^{\infty} d u \frac{1}{u^{2}+\frac{4 a c-b^{2}}{4 c}}
$$


Noting that $4 a c-b^{2}>0$, and using the standard integral [22]

$$
\int d u \frac{1}{u^{2}+\alpha^{2}}=\frac{1}{\alpha} \operatorname{ArcTan}\left(\frac{u}{\alpha}\right)
$$

we find finally

$$
T_{++}\left(\epsilon_{1}, \epsilon_{2}, \epsilon_{3}\right)=\frac{1}{4 \pi \sqrt{\left(\epsilon_{1}+\epsilon_{2}+\epsilon_{3}\right)}}\left(\pi+2 \operatorname{ArcTan}\left(\sqrt{\frac{\epsilon_{1} \epsilon_{3}}{\epsilon_{2}\left(\epsilon_{1}+\epsilon_{2}+\epsilon_{3}\right)}}\right)\right)
$$

Setting $\epsilon_{1}=\epsilon_{2}=\epsilon$ and $\epsilon_{3}=t-2 \epsilon$ it follows that

$$
g_{P}(0, t \mid 0,0)=\frac{1}{4}\left(1+\frac{2}{\pi} \operatorname{ArcTan}[(t-2 \epsilon) / t]^{\frac{1}{2}}\right)\left(\frac{m}{2 \pi i t}\right)^{\frac{1}{2}}
$$

for $2 \epsilon \leq t<3 \epsilon$, so we confirm the third equation in Eq.(4.2).

It will be useful for the three projection case below to record the following related results. A similar analysis to that above yields

$$
\begin{aligned}
T_{+-}\left(\epsilon_{1}, \epsilon_{2}, \epsilon_{3}\right)= & \int_{0}^{\infty} d y_{1} \int_{-\infty}^{0} d y_{2} \frac{1}{\pi \sqrt{\epsilon_{1} \epsilon_{2} \epsilon_{3}}} \exp \left(-\frac{y_{1}^{2}}{\epsilon_{1}}-\frac{\left(y_{1}-y_{2}\right)^{2}}{\epsilon_{2}}-\frac{y_{2}^{2}}{\epsilon_{3}}\right) \\
& =\frac{1}{4 \pi \sqrt{\left(\epsilon_{1}+\epsilon_{2}+\epsilon_{3}\right)}}\left(\pi-2 \operatorname{ArcTan}\left(\sqrt{\frac{\epsilon_{1} \epsilon_{3}}{\epsilon_{2}\left(\epsilon_{1}+\epsilon_{2}+\epsilon_{3}\right)}}\right)\right)
\end{aligned}
$$

In particular we then have

$$
\begin{aligned}
& T_{++}=T_{++}(\epsilon, \epsilon, \epsilon)=\frac{1}{3 \sqrt{3 \epsilon}} \\
& T_{+-}=\frac{1}{6 \sqrt{3 \epsilon}} \\
& T_{+0}=\frac{1}{2 \sqrt{3 \epsilon}}=\frac{1}{2} T_{00}
\end{aligned}
$$

We are able to evaluate the three projection case only in the situation where all time intervals are equal, so we set $t-n \epsilon=\epsilon$ in the definition of $T_{C}$ in Eq.(4.5). In this case, $T_{C}$ possesses a number of helpful symmetries. The first is "reflection" symmetry: if we define $-C$ by the string obtained by letting $(+\rightarrow-),(-\rightarrow+),(0 \rightarrow 0)$ then

$$
T_{-C}=T_{C}
$$

The second symmetry is "time reversal": if we define $\tilde{C}$ by the string obtained by reversing the order of $C$, then we have

$$
T_{\tilde{C}}=T_{C}
$$


In addition we have the simple property that

$$
T_{\ldots 0 \ldots}=T_{\ldots+\ldots}+T_{\ldots-\ldots}
$$

These properties imply the following for the three projection case. On the one hand we have,

$$
T_{+++}=T_{++0}-T_{++-}=T_{++0}-T_{0+-}+T_{-+-}
$$

but we also have,

$$
T_{+++}=T_{+0+}-T_{+-+}=T_{+0+}-T_{-+-}
$$

Combining these expressions gives,

$$
2 T_{+++}=T_{+0+}+T_{++0}-T_{0+-}
$$

For each of the objects on the right, we may carry out the full range integral, to leave ourselves with an object of the form $T_{C}\left(\epsilon_{1}, \epsilon_{2}, \epsilon_{3}\right)$ computed above. For example we have that $T_{+0+}=T_{++}(\epsilon, 2 \epsilon, \epsilon)$. Each of these objects may then be evaluated using Eq.(4.11) and Eq.(4.13), and combined to give,

$$
T_{+++}=\frac{1}{4 \sqrt{4 \epsilon}}
$$

It follows that

$$
g_{P}(0, t \mid 0,0)=\frac{1}{4}\left(\frac{m}{2 \pi i t}\right)^{\frac{1}{2}}
$$

when $t=4 \epsilon$, thus confirming the fourth equation in Eq.(4.2).

To end this section, we confirm our claim in Section 3 that the function $g_{P}(0, t \mid 0,0)$ drops to half its value immediately after a projection. On the face of it, this involves interpreting the expression $\langle 0| P$ which is ambiguous, so must be defined by a limiting procedure (where, recall $\langle 0|$ denotes $\langle x|$ at $x=0)$. We thus consider the limit $\epsilon_{n} \rightarrow 0$ in the expression

$$
\begin{aligned}
g_{P}(0, \tau \mid 0,0) & =\left\langle 0\left|e^{-i H \epsilon_{n}} P e^{-i H \epsilon} P \cdots e^{-i H \epsilon} P e^{-i H \epsilon_{0}}\right| 0\right\rangle \\
& =\int d y\left\langle 0\left|e^{-i H \epsilon_{n}} P e^{-i H \epsilon}\right| y\right\rangle\left\langle y\left|P \cdots e^{-i H \epsilon} P e^{-i H \epsilon_{0}}\right| 0\right\rangle
\end{aligned}
$$

We seek to show that the result obtained by this limit is half the result obtained when the final projection is absent. We write

$$
P=\frac{1}{2}(P+\bar{P})+\frac{1}{2}(P-\bar{P})
$$


so that

$$
\left\langle 0\left|e^{-i H \epsilon_{n}} P e^{-i H \epsilon}\right| y\right\rangle=\frac{1}{2}\left\langle 0\left|e^{-i H \epsilon_{n}} e^{-i H \epsilon}\right| y\right\rangle+\frac{1}{2}\left\langle 0\left|e^{-i H \epsilon_{n}}(P-\bar{P}) e^{-i H \epsilon}\right| y\right\rangle
$$

The first term on the right-hand side yields the sought after factor of a half as $\epsilon_{n} \rightarrow 0$, so it remains to show that the second term is zero. We have

$$
\left\langle 0\left|e^{-i H \epsilon_{n}}(P-\bar{P}) e^{-i H \epsilon}\right| y\right\rangle=\int_{0}^{\infty} d x\left\langle 0\left|e^{-i H \epsilon_{n}}\right| x\right\rangle\left(\left\langle x\left|e^{-i H \epsilon}\right| y\right\rangle-\left\langle-x\left|e^{-i H \epsilon}\right| y\right\rangle\right)
$$

where we have used the fact that

$$
\left\langle 0\left|e^{-i H \epsilon_{n}}\right| x\right\rangle=\left\langle 0\left|e^{-i H \epsilon_{n}}\right|-x\right\rangle
$$

(for the free particle, considered here). As $\epsilon_{n} \rightarrow 0,\left\langle 0\left|e^{-i H \epsilon_{n}}\right| x\right\rangle \rightarrow \delta(x)$ and the pair of terms in brackets on the right-hand side cancel in Eq.(4.28), so we obtain zero as required.

\section{ASYMPTOTIC LIMIT}

We now consider the asymptotic evaluation of $g_{P}(0, \tau \mid 0,0)$ for large $n$ in the case $\epsilon_{n}=$ $\epsilon=\epsilon_{0}$. This will give the values of the peaks of $f_{P}(t)$, Eq.(3.20).

Recall that in the limit $n \rightarrow \infty$ and $\epsilon \rightarrow 0$ with $\tau=(n+1) \epsilon$ fixed, $g_{P}\left(x_{1}, \tau \mid x_{0}, 0\right)$ tends to the restricted propagator $g_{r}\left(x_{1}, \tau \mid x_{0}, 0\right)$. To obtain the asymptotic form of $g_{P}(0, \tau \mid 0,0)$ for large $n$ we therefore need to determine the lowest non-trivial correction to this result. It is clear from the definition Eq.(3.2) of $g_{P}$ that close to the limit, we have the general form

$$
g_{P}\left(x_{1}, \tau \mid x_{0}, 0\right)=g_{r}\left(x_{1}, \tau \mid x_{0}, 0\right)+\epsilon g_{1}\left(x_{1}, \tau \mid x_{0}, 0\right)+O\left(\epsilon^{2}\right)
$$

for some function $g_{1}\left(x_{1}, \tau \mid x_{0}, 0\right)$. Since the restricted propagator vanishes if either $x_{1}=0$ or $x_{0}=0$, the object we need to calculate is

$$
g_{1}(0, \tau \mid 0,0)=\lim _{\epsilon \rightarrow 0, n \rightarrow \infty} \frac{g_{P}(0, \tau \mid 0,0)}{\epsilon}
$$

A standard and convenient way to do this is to rotate to imaginary time $\tilde{\tau}$ (we use tilde to denote Euclideanized time) and then write $g_{P}\left(x_{1}, \tilde{\tau} \mid x_{0}, 0\right)$ as a Euclidean path integral. This is then defined in terms of the continuum limit of probabilities of random walks on

a space time lattice of temporal spacing $\Delta \tilde{\tau}$ and spatial spacing $\eta$. The details of this construction are very conveniently given by Hartle [23] so we will give only the briefest 
account here. Using this language, $g_{P}(0, \tilde{\tau} \mid 0,0)$ is then the continuum limit of the object $(2 \eta)^{-1} u_{P}(0, \tilde{\tau} \mid 0,0)$, where $u_{P}(0, \tilde{\tau} \mid 0,0)$ is the probability for a random walk on the lattice starting at the origin and ending at the origin at time $\tilde{\tau}$, with the restriction that the walker is in the positive $x$-axis at the intermediate times $\tilde{\epsilon}, 2 \tilde{\epsilon}, 3 \tilde{\epsilon} \cdots($ where $\tilde{\epsilon} \geq \Delta \tilde{\tau})$.

The calculation of $u_{P}$ defined in this way, for values of $\tilde{\epsilon}$ generally greater than the lattice spacing $\Delta \tilde{\tau}$, is in fact a known problem in combinatorics called the tennis ball problem. It appears to have a formal solution, but this solution is too implicit for us to extract a useful result $[24]$.

Fortunately, however, for the purposes of calculating the limit Eq.(5.2) the results of Ref.[23] are sufficient. For this case, we set $\tilde{\epsilon}=\Delta \tilde{\tau}$ and $u_{P}(0, \tilde{\tau} \mid 0,0)$ is then the probability for a random walk from the origin to itself, with the restriction that the walker is in the positive $x$-axis at every intermediate step. On a finite lattice Hartle's calculations give the result,

$$
\frac{1}{2 \eta} u_{P}(0, \tilde{\tau} \mid 0,0)=\left(\frac{m}{2 \pi \tilde{\tau}}\right)^{1 / 2} \frac{\tilde{\epsilon}}{\tilde{\tau}}
$$

to leading order for small $\tilde{\epsilon}, \eta[23]$. We may use this result to compute the limit Eq.(5.2), which, continued back to real time, is

$$
g_{1}(0, \tau \mid 0,0)=\left(\frac{m}{2 \pi i \tau}\right)^{1 / 2} \frac{1}{\tau}
$$

Through Eq.(5.1), this confirms Eq.(3.20) for large $k$.

\section{A TIME AVERAGED RESULT}

We have argued that at the peak values of $g_{P}(0, \tau \mid 0,0)$ with $n$ projections, we have the approximate result

$$
\left\langle 0\left|e^{-i H \tau} P(n \epsilon) \cdots P(2 \epsilon) P(\epsilon)\right| 0\right\rangle \approx\left(\frac{m}{2 \pi i \tau}\right)^{1 / 2} \frac{1}{n+1}
$$

We showed above that this is exact for $n=1,2,3$ and true asymptotically for large $n$. Some interesting exact results for any $n$ may be obtained by considering a more general version of this object in which the projections are not restricted to act at the given set of times, $\epsilon, 2 \epsilon, 3 \epsilon \cdots$.

On the one hand, Eq.(3.17) may be expanded as a power series in powers of $V_{0}$ :

$$
g_{V}(0, \tau \mid 0,0)=\left(\frac{m}{2 \pi i \tau}\right)^{1 / 2} \sum_{n=0}^{\infty} \frac{(-1)^{n}}{(n+1) !} V_{0}^{n} \tau^{n}
$$


On the other hand, the evolution operator with complex potential may be expanded in the usual S-matrix expansion,

$$
\begin{aligned}
e^{-i(H-i V) \tau} & =e^{-i H \tau} T \exp \left(-\int_{0}^{\tau} d t V(t)\right) \\
& =e^{-i H \tau} \sum_{n=0}^{\infty} \frac{(-1)^{n}}{n !} \int_{0}^{\tau} d t_{n} \ldots \int_{0}^{\tau} d t_{1} T\left[V\left(t_{n}\right) \ldots V\left(t_{1}\right)\right]
\end{aligned}
$$

where $T$ denotes time ordering, $V(t)=V_{0} \bar{P}(t)$ and $\bar{P}(t)=e^{i H t} \bar{P} e^{-i H t}$. It follows that

$$
\begin{aligned}
g_{V}(0, \tau \mid 0,0) & =\langle 0|\exp (-i(H-i V) \tau)| 0\rangle \\
& =\sum_{n=0}^{\infty} \frac{(-1)^{n}}{n !} V_{0}^{n} \int_{0}^{\tau} d t_{n} \ldots \int_{0}^{\tau} d t_{1}\left\langle 0\left|e^{-i H \tau} T\left[\bar{P}\left(t_{n}\right) \ldots \bar{P}\left(t_{1}\right)\right]\right| 0\right\rangle
\end{aligned}
$$

Equating powers of $V_{0}$ in Eqs.(6.2) and (6.4) and writing out the time ordering explicitly, we deduce that

$$
\frac{n !}{\tau^{n}} \int_{0}^{\tau} d t_{n} \int_{0}^{t_{n}} d t_{n-1} \ldots \int_{0}^{t_{2}} d t_{1}\left\langle 0\left|e^{-i H \tau} \bar{P}\left(t_{n}\right) \ldots \bar{P}\left(t_{1}\right)\right| 0\right\rangle=\left(\frac{m}{2 \pi i \tau}\right)^{1 / 2} \frac{1}{n+1}
$$

We would like to write this in a form involving $P$, instead of $\bar{P}$. We introduce the reflection operator

$$
R=\int d x|x\rangle\langle-x|
$$

and note that $\bar{P}=R P R$, the Hamiltonian $H$ commutes with $R$, and $R|0\rangle=|0\rangle$ (where, recall, $|0\rangle$ denotes $|x\rangle$ at $x=0)$. It follows that

$$
\left\langle 0\left|e^{-i H \tau} \bar{P}\left(t_{n}\right) \ldots \bar{P}\left(t_{1}\right)\right| 0\right\rangle=\left\langle 0\left|e^{-i H \tau} P\left(t_{n}\right) \ldots P\left(t_{1}\right)\right| 0\right\rangle
$$

so that Eq.(6.5) holds with all the $\bar{P}$ 's replaced with $P$ 's. Noting that

$$
\frac{n !}{\tau^{n}} \int_{0}^{\tau} d t_{n} \int_{0}^{t_{n}} d t_{n-1} \cdots \int_{0}^{t_{2}} d t_{1}=1
$$

we see that Eq.(6.5) is of the desired general form, Eq.(6.1), but time-averaged over the times of the projections.

The question is now to what extent the time-averaged expression on the left-hand side of Eq.(6.5) is close to Eq.(6.1). We may take this further in two different ways.

First, note that for a real-valued function $f$ of $n$ variables, we have the mean value theorem

$$
\frac{n !}{\tau^{n}} \int_{0}^{\tau} d t_{n} \int_{0}^{t_{n}} d t_{n-1} \cdots \int_{0}^{t_{2}} d t_{1} f\left(t_{n}, t_{n-1} \cdots t_{1}\right)=f\left(\xi_{n}, \xi_{n-1} \cdots \xi_{1}\right)
$$


for some set of numbers $\xi_{n} \geq \xi_{n-1} \geq \cdots \geq \xi_{1}$ in the interval $[0, \tau]$. The integral in Eq.(6.5) is easily made into a real integral over a real-valued function by analytic continuation, and we therefore deduce the exact result

$$
\left\langle 0\left|e^{-i H \tau} P\left(\xi_{n}\right) \ldots P\left(\xi_{1}\right)\right| 0\right\rangle=\left(\frac{m}{2 \pi i \tau}\right)^{1 / 2} \frac{1}{n+1}
$$

for some set of projection times $\xi_{n} \geq \xi_{n-1} \geq \cdots \geq \xi_{1}$ in the interval $[0, \tau]$.

Second, we may expand the integrand in the left-hand side of Eq.(6.5) about the values $k \epsilon$, to get some insight into why these particular values have any special significance. We have

$$
\begin{aligned}
\left\langle 0\left|e^{-i H \tau} P\left(t_{n}\right) \ldots P\left(t_{1}\right)\right| 0\right\rangle & =\left\langle 0\left|e^{-i H \tau} P(n \epsilon) \ldots P(\epsilon)\right| 0\right\rangle \\
& +\left.\sum_{k=1}^{n}\left(t_{k}-k \epsilon\right) \frac{\partial}{\partial t_{k}}\left\langle 0\left|e^{-i H \tau} P\left(t_{n}\right) \ldots P\left(t_{1}\right)\right| 0\right\rangle\right|_{t_{k}=k \epsilon} \\
& +\cdots
\end{aligned}
$$

Inserting in Eq.(6.5), and noting that

$$
\frac{n !}{\tau^{n}} \int_{0}^{\tau} d t_{n} \int_{0}^{t_{n}} d t_{n-1} \cdots \int_{0}^{t_{2}} d t_{1} t_{k}=k \epsilon
$$

(where, recall, $\tau=(n+1) \epsilon$ ) we see the first order term in the expansion Eq.(6.11) averages to zero in Eq.(6.5). This is clearly only true for expansion about the special values $t_{k}=k \epsilon$. We therefore obtain the desired result Eq.(6.1) up to second order corrections. This suggests that the values $t_{k}=k \epsilon$ are significant because they give the best approximation to the average in Eq.(6.5). These results give evidence that Eq.(3.20) holds approximately for all $n$, including the intermediate values not covered in the previous two sections.

\section{NUMERICAL RESULTS}

To support the analytic results described in the previous sections we evaluate Eq.(3.18) numerically for up to 20 projections and confirm the conjectured form Eq.(3.19), (3.21). It is convenient to define a sequence of functions $F_{n}(t, x)$ defined by

$$
F_{0}(t, x)=\left\langle x\left|e^{-i H t}\right| 0\right\rangle
$$

for $0 \leq t<\epsilon$ and

$$
F_{n}(t, x)=\left\langle x\left|e^{-i H(t-n \epsilon)}\left(P e^{-i H \epsilon}\right)^{n}\right| 0\right\rangle
$$


for $n \epsilon \leq t \leq(n+1) \epsilon$ where $n=1,2,3 \cdots$. The desired object Eq.(3.18) (with, for convenience, $\left.\epsilon_{0}=\epsilon\right)$, is then given by

$$
g_{P}(0, t \mid 0,0)=F_{n}(t, 0)
$$

The sequence $F_{n}(t, x)$ may be calculated using the recursion relation,

$$
F_{n}(t, x)=\int_{0}^{\infty} d y\left\langle x\left|e^{-i H(t-n \epsilon)}\right| y\right\rangle F_{n-1}(n \epsilon, y)
$$

Using a new time coordinate $s$ defined by $t=s \epsilon$, rotating to imaginary time, and defining $\tilde{F}_{n}(s, x)=F_{n}(-i t, x)$, we have

$$
\tilde{F}_{n}(s, x)=\left(\frac{m}{2 \pi(s-n)}\right)^{\frac{1}{2}} \int_{0}^{\infty} d y \exp \left(-\frac{m(x-y)^{2}}{2(s-n)}\right) \tilde{F}_{n-1}(n, y)
$$

for $n \leq s \leq n+1$.

We have attempted to find an approximate analytic solution to Eq.(7.5) for large $n$, but without success. However, a numerical solution is straightforward and yields all the information we require. This was done using a simple mid-point rule. The lattice size was chosen to be of order $10^{-3}$, and the results were checked for robustness against changes in lattice size.

The numerical result for $f_{P}(t)$ (defined in Eq.(3.19) in terms of $g_{P}(0, t \mid 0,0)$ ) is plotted in Figure 3, along with our claimed approximate analytic expression for $f_{P}(t)$, Eq.(3.21). We see that there is excellent agreement. The values at the peaks and troughs appear to agree perfectly. The only small discrepancy is that the interpolating functions between the peaks and troughs are not exactly linear. This discrepancy is only noticeable for intermediate values of $n$ and in any event since, as argued, the curve is effectively averaged over time in the PDX, this discrepancy is insignificant. We therefore find substantial numerical confirmation for our our main result, Eqs.(3.19), (3.21).

The apparently perfect agreement of numerical results with the approximate analytic expression Eq.(3.21) at the peaks and troughs is striking. We wonder if the approximate analytic expression is in fact exact at these points, but we have not been able to prove this, except for the cases $n=1,2,3$ and for large $n$.

A useful way of seeing even more precisely the relationship between $f_{P}(t)$ and $f_{V}(t)$ is to define the function

$$
S(t)=\frac{f_{P}(t)}{f_{V}(t)}-1
$$


so that

$$
f_{P}(t)=(1+S(t)) f_{V}(t)
$$

This is plotted in Figure 4. It is a simple function oscillating around zero between $\pm 1 / 3$ with period $\epsilon$. In terms of $S(t)$, the relationship between $g_{P}$ and $g_{V}$ then has the particularly simple form

$$
g_{P}(0, t \mid 0,0)=(1+S(t)) g_{V}(0, t \mid 0,0)
$$

This relationship is perhaps the most concise summary of the sought-after connection between the propagators.

\section{TIMESCALES}

We now give a more detailed explanation as to the timescales involved in proving the approximate equivalence of $g_{V}$ and $g_{P}$. We broadly expect that the appropriate timescale is the Zeno time of the initial state, $t_{Z}=1 / \Delta H$. However, we have derived a very precise connection between evolution in the presence of a complex potential and evolution with projection operators so we are in a position to investigate the specific way in which an initial state may discriminate between these two types of evolution.

We have argued that the equivalence boils down to proving the equivalence of propagation along the boundary, Eq.(3.12), and we have shown that $g_{P}(0, t \mid 0,0)$ oscillates around $g_{V}(0, t \mid 0,0)$ with period $\epsilon$. Suppose we have an initial state $\psi(x, 0)$. Let us consider the change in the wave function arising from replacing $g_{V}$ with $g_{P}$ using the PDX, Eq.(3.10). It is

$$
\delta \psi\left(x_{1}, \tau\right)=\left.\frac{1}{m^{2}} \int_{0}^{\tau} d t_{2} \int_{0}^{t_{2}} d t_{1} \frac{\partial g_{f}}{\partial x}\left(x_{1}, \tau \mid x, t_{2}\right)\right|_{x=0} \delta g\left(0, t_{2} \mid 0, t_{1}\right) \frac{\partial \psi}{\partial x}\left(0, t_{1}\right)
$$

where

$$
\delta g\left(0, t_{2} \mid 0, t_{1}\right)=g_{V}\left(0, t_{2} \mid 0, t_{1}\right)-g_{P}\left(0, t_{2} \mid 0, t_{1}\right)
$$

and we also used Eq.(3.11). The important part of this expression is the $t_{1}$ integral, which we expect will be small if the initial state is sufficiently slowly varying in time.

The results of Section 3 and 8 show that $\delta g(0, t \mid 0,0)$ oscillates around zero with period $\epsilon$. In particular, Eq.(7.8) shows that

$$
\delta g(0, t \mid 0,0)=S(t) g_{V}(0, t \mid 0,0)
$$


The explicit form of $S(t)$ is given in Figure 4, but its important qualitative feature is its oscillation with period $\epsilon$, so for simplicity, $S(t)$ may be loosely modelled by the function $\sin (2 \pi t / \epsilon)$. To be definite we take the initial state $\psi$ to be a Gaussian wave packet, so we have

$$
\psi(x, t)=N \exp \left(-\frac{(x-q-p t / m)^{2}}{4 \sigma^{2}}+i p x-i E t\right)
$$

where $E=p^{2} / 2 m, N$ is a normalization factor, and we have ignored wave packet spreading effects in evolving the state. For such a state the Zeno time is $t_{Z}=m \sigma / p$. In Eq.(8.1) the differentiation of $\psi$ produces a prefactor which does not contribute to the leading order evaluation of the time integral, and if the range of integration is much greater than $\epsilon$, we obtain the order of magnitude result

$$
\int d t_{1} \delta g\left(0, t_{2} \mid 0, t_{1}\right) \frac{\partial \psi}{\partial x}\left(0, t_{1}\right) \sim \exp \left(-\frac{t_{Z}^{2}}{\epsilon^{2}}(E \epsilon-1)^{2}\right)
$$

If $E \epsilon \ll 1$, the right-hand side is clearly very small if $\epsilon \ll t_{Z}$. If $E \epsilon>2$, the right-hand side if bounded from above by $\exp \left(-t_{Z}^{2} / \epsilon^{2}\right)$ so again will be small if $\epsilon \ll t_{Z}$. Hence the Zeno time of the initial state is indeed the timescale controlling the validity of the approximation, as expected. Note also that Eq.(8.5) goes to zero as $\epsilon \rightarrow 0$, as it must, since $g_{P}$ and $g_{V}$ become exactly equal (and equal to $g_{r}$ ) in this limit.

The only problematic case is that in which the initial state has energy $E \sim 1 / \epsilon$. In this case, the right-hand side of Eq.(8.5) is not necessarily small and the approximation may fail. This is not surprising since it is the case in which the oscillations in time of the incoming state are comparable to the temporal spacing of the projections, so that the state can "see" the difference between the complex potential and projections at a discrete set of times.

Hence, apart from the above exception, Eq.(3.12) holds for $\epsilon \ll t_{Z}$. As outlined in the Introduction, reflection in the complex potential is negligible if $V_{0} \ll E$ which is equivalent to $\epsilon \gg 1 / E$. Therefore there is an interesting regime, namely

$$
\frac{1}{E} \ll \epsilon \ll \frac{1}{\Delta H}
$$

in which the approximate equivalence Eq.(3.12) holds, yet there is negligible reflection. This regime is important in, for example, study of the arrival time problem using complex potentials [9]. This issue will considered in more detail elsewhere [26]. (See also Ref.[25] for an interesting discussion of timescales in the Zeno effect.) 


\section{SUMMARY AND DISCUSSION}

This paper was physically motivated by the desire to understand the effect of periodically acting projections onto the positive $x$-axis for a free particle, Eq.(1.1). A valuable way to proceed is to use the conjectured relationship Eq.(1.3) with a complex potential first put forward by Echanobe et al. This connection, together with known results on scattering, establishes the timescale under which significant reflection occurs in Eq.(1.1). We noted that the arguments for the relationship Eq.(1.3) are only heuristic and there is scope for a more substantial proof.

We proved Eq.(1.3) by considering the associated propagators Eqs.(3.1), (3.2). We noted that an approximate equivalence between these propagators is expected since both propagators tend to the restricted propagator $g_{r}\left(x_{1}, \tau \mid x_{0}, 0\right)$ in the limits $\epsilon \rightarrow 0, n \rightarrow \infty$ and $V_{0} \rightarrow \infty$. The path decomposition expansion reduced the proof of equivalence of these propagators to the simpler case of propagation between points lying on the origin, Eq.(3.12). The propagator along the origin for the complex potential $g_{V}(0, t \mid 0,0)$ is already known, Eq.(3.17), so the bulk of the proof was to derive the analogous result for the propagator with projections, $g_{P}(0, \tau \mid 0,0)$. Our main result was to prove that this propagator has the approximate form Eqs.(3.19), (3.21), which we proved using a variety of analytic and numerical methods. In effect, the main achievement of this paper has therefore been to obtain a good approximate analytic expression for the propagator $g_{P}$ that appears in Eq.(1.1).

We found that $g_{P}(0, t \mid 0,0)$ oscillates with period $\epsilon$ around $g_{V}(0, t \mid 0,0)$ as long as $V_{0} \epsilon \approx$ 4/3, a result most concisely summarized in Eq.(7.8). The approximate equivalence Eq.(3.12) of these propagators then holds in a time-averaged sense as long as the timescale $\epsilon$ between projections is much smaller than the Zeno time of the initial state, $1 / \Delta H$ (but may fail in the special case when the incoming state is peaked about energy $E \sim 1 / \epsilon$ ). These conditions agree in essence with those of an extended version of the results of Echanobe et al, with an advantage over their results that a definite relationship between $V_{0}$ and $\epsilon$ is obtained. We noted that their restriction Eq.(1.4) relating $V_{0}$ and $\epsilon$ is in fact stronger than required and

the equality $V_{0} \epsilon \approx 4 / 3$ derived here gives the best approximate equivalence between $g_{P}$ and $g_{V}$.

In addition to helping establish the timescales for reflection in Eq.(1.1), the connection Eq.(1.3) has been of use in investigating the near-Zeno regime of Eq.(1.1) [14-16]. In the 
limit $\epsilon \rightarrow 0$, the Zeno limit, motion in Eq.(1.1) is confined entirely to states with support only in $x>0$ and is described by the restricted propagator Eq.(3.8). It is of interest to explore the nature of the dynamics very close to this regime, i.e., for small but finite $\epsilon$. This can be achieved by examining the form of the propagator with complex potential $g_{V}$ for large $V_{0}$. In this regime, one can calculate, for example, the (unnormalized) probability density for crossing the origin between $\tau$ and $\tau+d \tau$, which is found to be

$$
\begin{aligned}
\Pi(\tau) & =-\frac{d N}{d \tau} \\
& =\frac{2}{m^{3 / 2} V_{0}^{1 / 2}}\left\langle\psi_{f}(\tau)|\hat{p} \delta(\hat{x}) \hat{p}| \psi_{f}(\tau)\right\rangle
\end{aligned}
$$

Here, $N(\tau)$ is the survival probability (given by the norm of the state evolved in the presence of the complex potential) and $\left|\psi_{f}(\tau)\right\rangle$ is the freely evolved wave function. This is proportional to the average kinetic energy density at the origin. As anticipated it goes to zero as $V_{0} \rightarrow \infty$ since there is total reflection. However, it is useful to define a normalized distribution,

$$
\begin{aligned}
\Pi_{N}(\tau) & =\frac{\Pi(\tau)}{\int_{0}^{\infty} d t \Pi(t)} \\
& =\frac{1}{m\langle p\rangle}\left\langle\psi_{f}(\tau)|\hat{p} \delta(\hat{x}) \hat{p}| \psi_{f}(\tau)\right\rangle
\end{aligned}
$$

where $\langle p\rangle$ is the average momentum in the initial wave packet [14-16]. Interestingly, this is now independent of the complex potential despite this being the regime of strongly-acting measurement. This regime will be explore further in future publications [26].

Finally, we comment on the possible generality of the connection Eq.(1.3). We first note that it may in fact be written

$$
e^{-i H \epsilon} P e^{-i H \epsilon} \cdots P e^{-i H \epsilon} \approx \exp \left(-i H \tau-V_{0} \bar{P} \tau\right)
$$

where $\bar{P}=1-P$. We have proved Eq.(9.3) for the case in which the projections are onto the positive $x$-axis, but it seems reasonably clear that the relationship will hold for projections onto any region $[a, b]$ of the $x$-axis (as long as it is not too small) and indeed for regions of configuration space in a many-dimensional model. Such a potential has been used recently in the decoherent histories analysis of quantum cosmological models [27]). Moreover, although the proof of Eq.(9.3) given in this paper relied heavily on the fact that $P$ projects onto position, the form Eq.(9.3) and the heuristic argument for it in Section 2 do not rely on the particular form of $P$. We therefore conjecture that Eq.(9.3) may hold for a wider variety of projection operators, not just projectors onto position. This will be pursued elsewhere. 


\section{Acknowledgments}

We are grateful to Carl Bender for useful discussions and to Adolfo del Campo for helpful comments on the first draft of this paper.

[1] M.Gell-Mann and J.B.Hartle, in Complexity, Entropy and the Physics of Information, SFI Studies in the Sciences of Complexity, Vol. VIII, W. Zurek (ed.) (Addison Wesley, Reading, 1990); and in Proceedings of the Third International Symposium on the Foundations of Quantum Mechanics in the Light of New Technology, S. Kobayashi, H. Ezawa, Y. Murayama and S. Nomura (eds.) (Physical Society of Japan, Tokyo, 1990).

[2] M.Gell-Mann and J.B.Hartle, Phys.Rev. D47, 3345 (1993).

[3] R.B.Griffiths, J.Stat.Phys. 36, 219 (1984); Phys.Rev.Lett. 70, 2201 (1993); Am.J.Phys. 55, $11(1987)$.

[4] R.Omnès, J.Stat.Phys. 53, 893 (1988); 53, 933 (1988); 53, 957 (1988); 57, 357 (1989); 62, 841 (1991); Ann.Phys. 201, 354 (1990); Rev.Mod.Phys. 64, 339 (1992); The Interpretation of Quantum Mechanics (Princeton University Press, Princeton, 1994).

[5] J.J.Halliwell, in Fundamental Problems in Quantum Theory, edited by D.Greenberger and A.Zeilinger, Annals of the New York Academy of Sciences, Vol 775, 726 (1994).

[6] H.F.Dowker and J.J.Halliwell, Phys. Rev. D46, 1580 (1992).

[7] J.G.Muga, R.Sala Mayato and I.L.Egusquiza (eds), Time in Quantum Mechanics (Springer, Berlin, 2002); J.G.Muga and C.R.Leavens, Phys.Rep.338, 353 (2000).

[8] G.R.Allcock, Ann.Phys 53, 253 (1969); 53, 286 (1969); 53, 311 (1969).

[9] J.J.Halliwell and J.M.Yearsley, Phys. Rev. A 79, 062101 (2009).

[10] J.J.Halliwell and J.M.Yearsley, Phys. Lett. A374, 154-157 (2009).

[11] B.Misra and E.C.G.Sudarshan, J.Math.Phys. 18, 756 (1977); A. Peres, Am. J. Phys. 48, 931 (1980).

[12] P.Wallden, Int.J.Theor.Phys.47, 1512 (2008).

[13] P. Facchi, S. Pascazio, A. Scardicchio, L. S. Schulman, Phys. Rev. A 65, 012108 (2002).

[14] J.Echanobe, A. del Campo and J.G.Muga, Phys. Rev. A 77, 032112 (2008).

[15] J.G.Muga, D.Seidel and G.C.Hegerfeldt, J.Chem.Phys. 122, 154106 (2005). 
[16] J.J.Halliwell, Phys.Rev. A77, 062103 (2008).

[17] J.G.Muga, S.Brouard, D.Macias, Ann. Phys. (NY) 240, 351 (1995); Ph.Blanchard and A.Jadczyk, Helv. Phys. Acta 69, 613 (1996); J.P.Palao, J.G.Muga, S.Brouard, A.Jadczyk, Phys. Lett. A233, 227 (1997); A.Ruschhaupt, J.Phys. A35, 10429 (2002); J.G. Muga, J.P. Palao, B. Navarro, I.L. Egusquiza, Phys. Rep. 395, 357 (2004); J.J.Halliwell, Prog.Th.Phys.102, 707 (1999).

[18] A.Auerbach and S.Kivelson, Nucl.Phys. B257, 799 (1985); P. van Baal, in Lectures on Path Integration, edited by H.A. Cerdeira et al (World Scientific, Singapore, 1993) [also available from the website http://www.lorentz.leidenuniv.nl/vanbaal/HOME/publ.html];

[19] J.J.Halliwell and M.E.Ortiz, Phys.Rev. D48, 748 (1993).

[20] J.J.Halliwell, Phys.Lett. A207, 237 (1995).

[21] T.O. de Carvalho, Phys. Rev. A47, 2562 (1993); J.M.Yearsley, J. Phys. A 41, 285301 (2008).

[22] I.S.Gradshteyn and I.M.Ryzhik, Table of Integrals, Series and Products, Fifth Edition (Academic Press, Boston, 1994).

[23] J.B.Hartle, Phys.Rev. D37, 2818 (1988).

[24] A. de Mier and M. Noy, arXiv:maths/0311242 (2003).

[25] L.Schulman, in Time in Quantum Mechanics, edited by J.G.Muga, R.Sala Mayato and I.L.Egusquiza (Springer, Berlin, 2002) (also available as arXiv:quant-ph/0103151).

[26] J.M.Yearsley, in preparation.

[27] J.J.Halliwell, Phys. Rev. D80, 124032 (2009). 


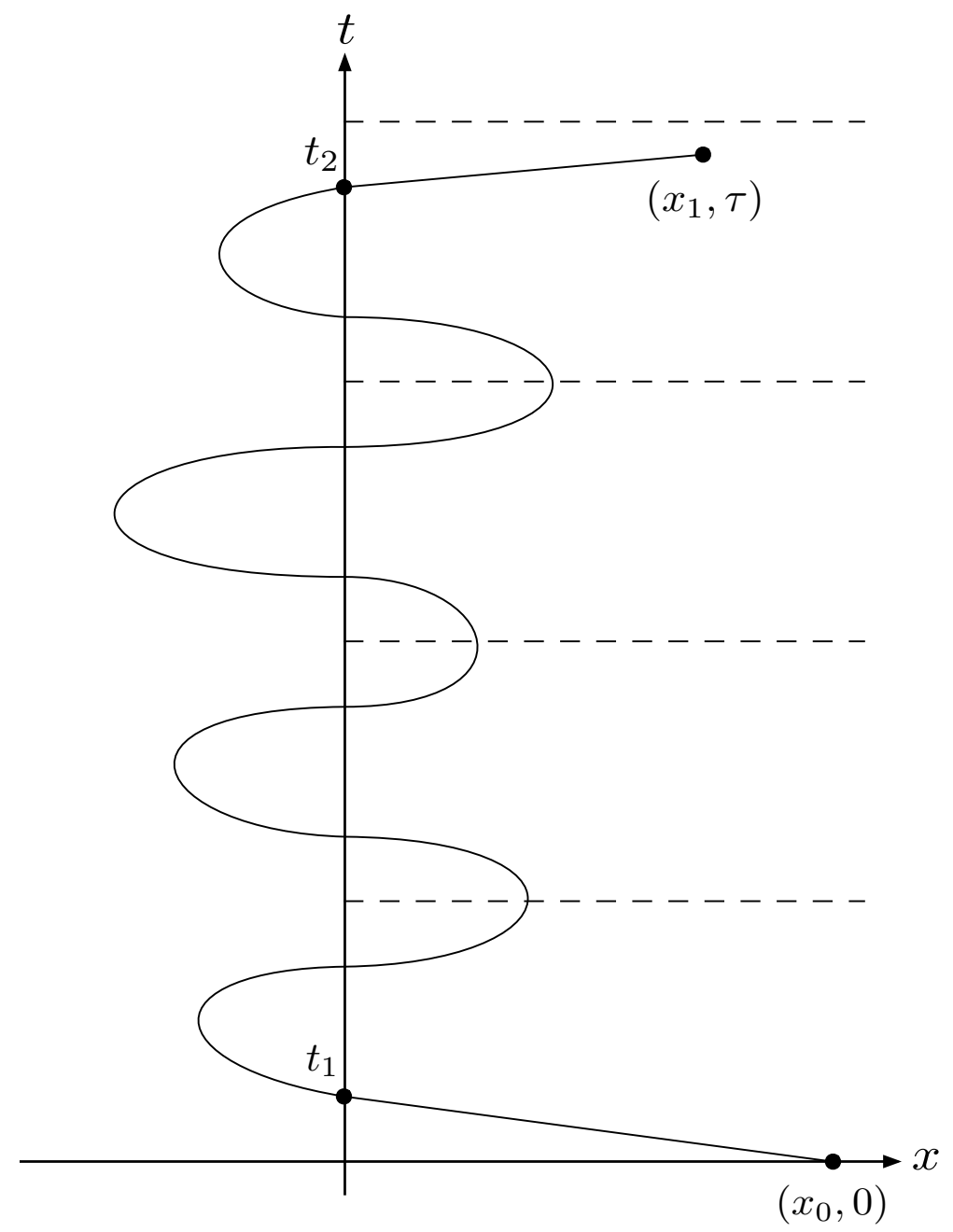

Figure 1. The path decomposition expansion for $g_{P}$. Any path from $x_{0}>0$ at $t=0$ to a final point $x_{1}>0$ at $t=\tau$ which crosses $x=0$ has a first crossing of $x=0$ at $t_{1}$ and a last crossing at $t_{2}$. The crossing part of the propagator from $\left(x_{0}, 0\right)$ to $\left(x_{1}, \tau\right)$ may therefore be decomposed into three parts: (A) restricted propagation entirely in $x>0$, (B) propagation starting and ending on $x=0$ with the restriction that the paths are in $x>0$ at a discrete set of times, and $(\mathrm{C})$ restricted propagation entirely in $x>0$. The corresponding path decomposition expansion formula is given in Eq.(3.10). (The $g_{r}$ term corresponds to paths which never cross $x=0$ ). 


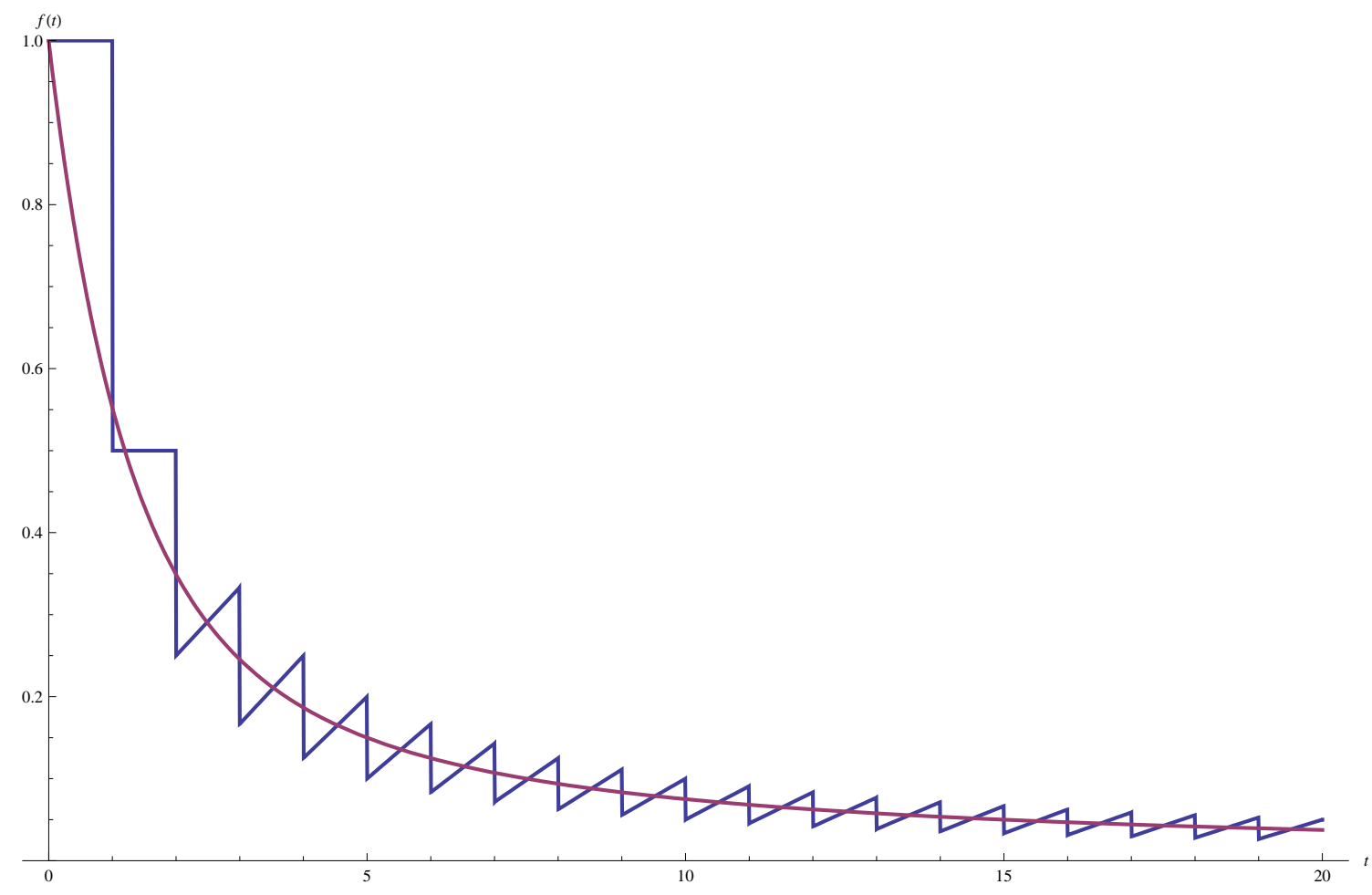

Figure 2. A plot of the functions $f_{V}(t)$ and $f_{P}(t)$, defined for the complex potential by Eq.(3.17) with $V_{0} \epsilon=4 / 3$, and for intermittent projectors by Eqs.(3.19), (3.21). (The time scaling is such that $\epsilon=1$ in the plot, so that the peaks occur at integer values of $t$ ). We see that $f_{P}(t)$ oscillates around $f_{V}(t)$ with period $\epsilon$ so the two functions are equal in a timeaveraged sense, when integrated against functions which are slowly varying on a timescale $\epsilon$. 


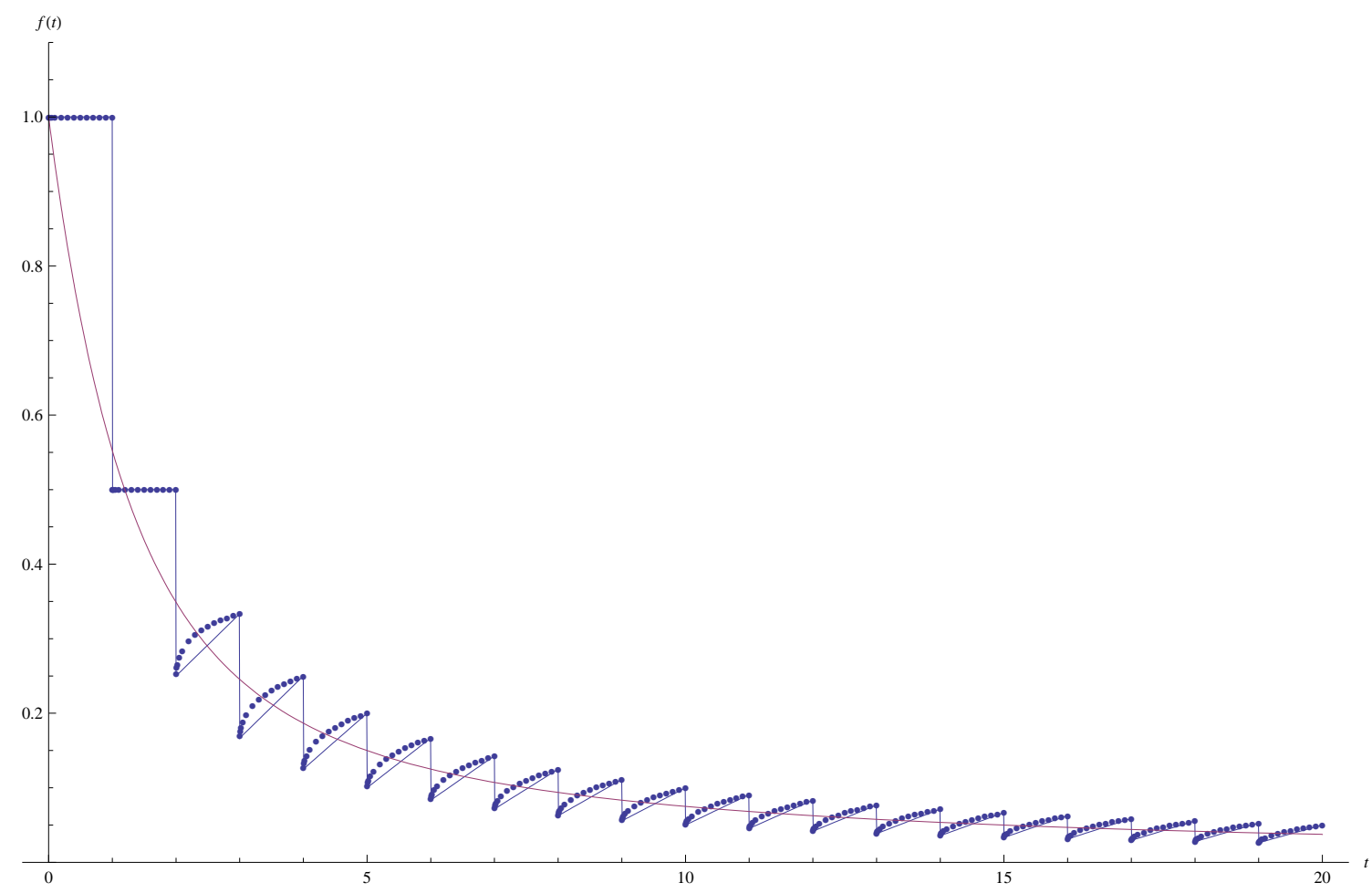

Figure 3. A plot of the numerical calculation of $f_{P}(t)$ (the set of dotted lines), and the conjectured analytic expression for $f_{P}(t)$, Eqs.(3.19), (3.21) (the set of straight lines). (Again we use a time scaling such that $\epsilon=1$ ). There is excellent agreement between analytic and numerical results at the peaks and troughs of the functions. The numerical result shows some departure from exact linear behaviour between the peaks and troughs but this is insignificant as argued in the main text. A plot of $f_{V}(t)$, the smooth curve, is also given for reference. 


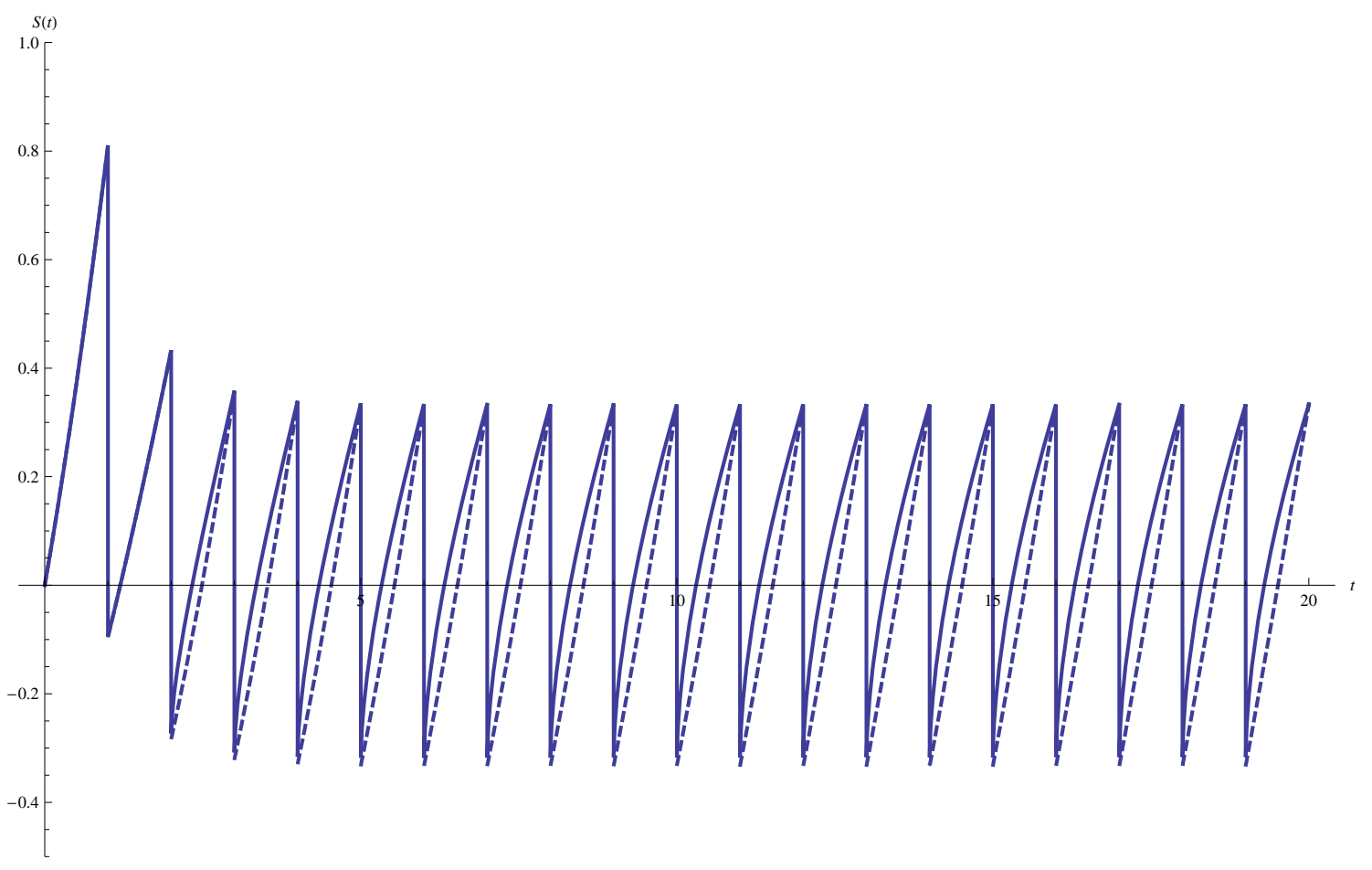

Figure 4. A plot of the function $S(t)$ defined in Eq.(7.6), describing the oscillations of $g_{P}(0, t \mid 0,0)$ around $g_{V}(0, t \mid 0,0)$. The bold line represents the numerical calculation and the dashed line the analytic result (from Eqs.(3.17), (3.21)). After the first few oscillations, it oscillates between $\pm 1 / 3$ (approximately). 